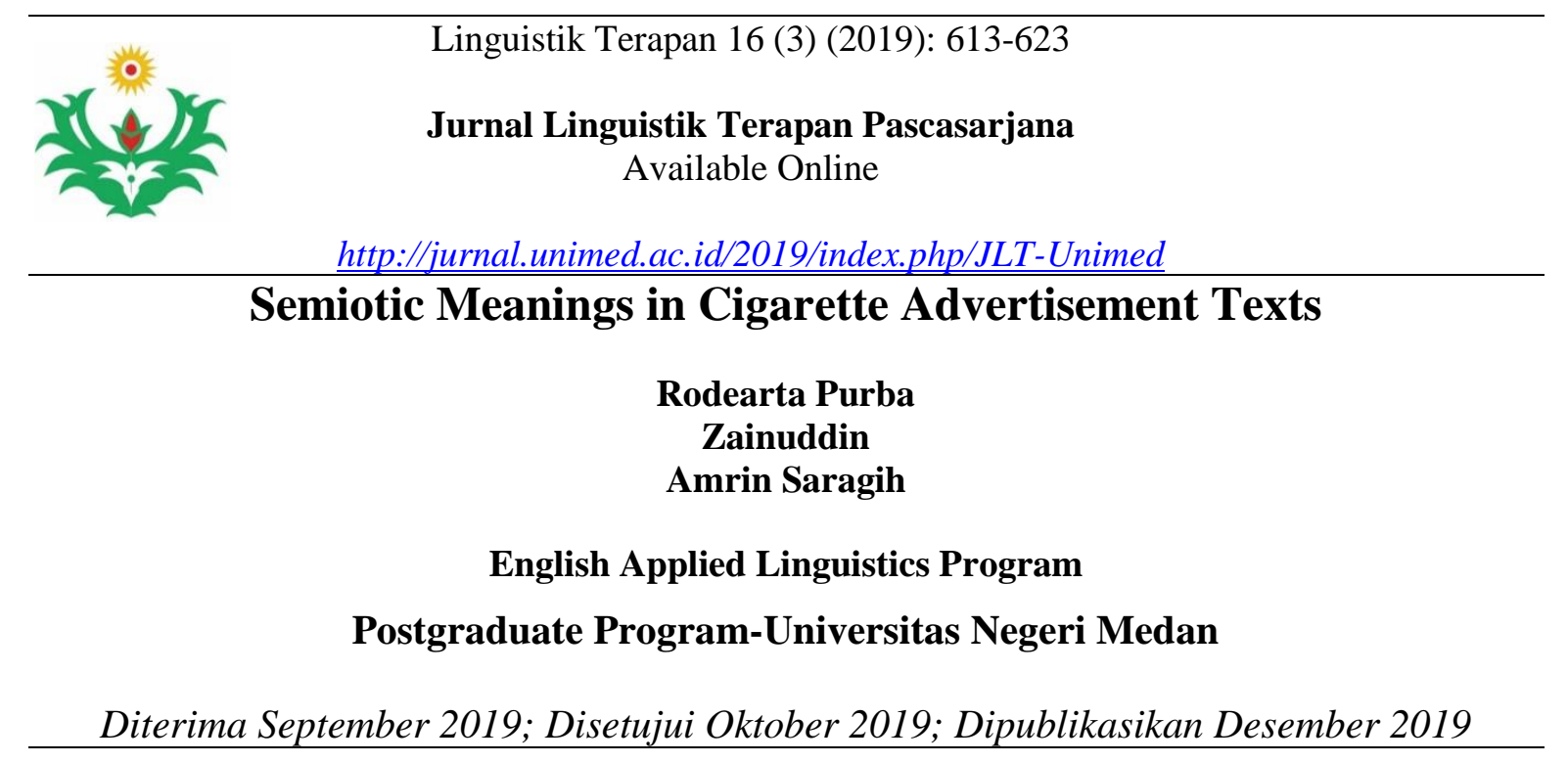

\begin{abstract}
Semiotic is the study of sign or the science that study about the sign in human beings. The objectives of this study were to investigate the semiotic meanings applied in cigarette advertisement, to analyze how the meanings were coded verbally and non-verbally, and to reason why it is used in the ways they are. The data for the study were clauses, phrases, and sentences drawn from selected billboard advertisement of cigarette in Medan. The verbal data analyzed with linguistic devises and textual analysis of Leech Theory and visual grammar of Kress and Van Leeuwen as theoretical framework. The result were two broad categorize of meaning. They were related to verbal and non-verbal aspects. The relation between the verbal and non-verbal expression were elaborate, manipulate, and exageratte, the advertisers make the major use of declarative sentence that are found on 11 texts. Then, by using 8 imperative and 8 adjective. Next, by using 6 disjunctive and 6 noun/pronoun. In image/picture use of visual features such as calor saturation, symbolic structure representation, offering gaze, the use of large size, logo, slogan. Various social context were discoverd in the advertisement such as promoting the standar of quality, quantity, creative, enjoying the life. The standard idea about how particular mode like young and old man should be differentiated based on the conventional values in society when it comes to what types of product that is competible to them. Cigarette advertisement also hide threats with persuasive expression and colorful meaning for commercial purposes.
\end{abstract}

Keywords: Semiotics, Sign, Critical Discourse Analysis, Cigarette Advertisement.

How to Cite: Purba, Rodearta (2019). Semiotic Meanings in Cigarette Advertisement Texts. Jurnal Linguistik Terapan Pascasarjana Unimed.

ISSN 0216-5139

\title{
INTRODUCTION
}

As a form of mass communication, advertising is closely link with the world of commerce and marketing. Advertising, therefore, is a powerful tool for the flow of information from sellers to buyers. It does not only influence and persuade people to act or 
believe but also reflects certain aspect of the society's value and structure. In this case, mass media, like newspaper, megazine, television, radio, internet, etc, is used to reach the mass amount of people. In all media, both electronic and print, information materials of all kinds and texts in a general sense, there is a complex network of written text accompanied by images and other non-linguistic elements, designed as coherent entities (they are often more visual than verbal) 'by the means of layout'(Kressand Leeuwen 2006: 17). Language also can be seen as a place of exchange of meaning. The message conveyed in the text will interact with readers so that the meaning produced.

Essentially, advertising is a sign that implied by the producer to the consumer to be, considered and subsequently taken into consideration to make a decision to purchase (use) or not a product. Keraf (1992: 3) stated that a way of using language as an art, both oral and written, is based on a well structured knowledge for the purpose of influencing the attitudes and feelings of others. Life style provides an opportunity for manufacturers to compete to offer their products, they are trying to mobilize all the expertise, creativity in advertising create and use a language th at is attractive and convincing in order to lure consumers. The choosing of words, colors, and images become the main point as the tool to persuade the listener and the readers to buy their product or to use their service. Seeing from the perspective of advertisement, cigarette product also tries to persuade people to accept and puchase their product. To do so, advertiser uses linguistic aspect, pictorial aspect, music aspect and etc. This is to influence the mind of people to buy their product.

However, cigarette advertisements are different from others. The differences of cigarette advertisement is that it does not show detail information of their product. This is because the regulation of government dealing with cigarette advertisement which is say that cigarette companies have forbidden to show the package of cigarette, smoking people, smokes and even information about the product.

A review of main literature on critical discourse analysis and terminology of visual semiotic analysis, and supporting theories of advertisement related to this study are presented in this chapter. The verbal text analyzed based on Fairclough theory $(1995,2001,2006)$. It is about three level approach of critical discourse analysis a tool for data analysis, and for the non-verbal (visual semiotic analysis), it was anlyzed based on Kress and van Leeuwen theory (2006).

\section{Critical Discourse Analysis}

Critical Discourse Analysis (CDA) sees language as social and considers the context of language use to be crucial. CDA is not restricted to the educational institution. Discourse Analysis (DA) studies discourse from linguistics perspectives that structural oriented 
analysis such as implicatures, turn taking, speech acts, politeness, etc. CDA look at discourse not only from linguistic perspective but also goes beyond the boundary of textgrammar toward a study of action-interaction (Huckin, 2007). CDA is therefore not interested in investigating a linguitic unit as DA does, but in studying social phenomena which are necessarily complex and thus require multidisiplinary and multi-methodical approach (Wodack and Meyer, 2008). CDA sstudies any types of discourses (written, spoken, and semiotic) which can systematically communicate with us. CDA tends to establish a relationship between language (semiotic signs, paralanguage, advertisements, etc), ideology and power (Huckin, 2007: 2-3).

The aim of CDA is to inform the people about the hidden problems of society so that they can ask questions about the hidden the hidden issues. (Huckin: 2007). CDA related to this study not only limited t a vast number phenomena of verbal or linguistic aspects but also pay attention to non-verbal and semiotic aspect.

Based on explanation above, CDA does not only studies language use that found in advertisement but also another type of discourse such as semiotic that related to advertisement which has been the writer's focus in this research.

\section{Semiotics}

Semiotics is the study of sign or the science that study about the sign in human beings. It means, every exist things in our life is looked as the sign, that is something that we should give the meaning. Semiotisc is concerned with everything that can be taken as sign. A sign is everything which can be taken as significantly substituting for something else.

The visual design is as equally important as the verbal text. Its analysis should potentially provide evidence for answering the research questions of this study. Consering the use of image in advertisements, more technical terms for describing various aspects of images are discussed by Scollon and Scollon (2003) and Kress and Van Leeuwen (2006) in this session.

The use of the model of discourse of Kress and Van Leeuwen in this study is limited because the writer does not apply all of its components. The semiotic devices used in this study are mainly based on some of the following of its components as summarizedd by Scollon and Scollon (2003): conceptual representation, gaze/contact, social distance, modality of color, salience, and information values. These categories could not be used uniformly on all text because each text has its own peculiar characteristi 


\section{METHODOLOGY}

This research was conducted by using descriptive qualitative design. The data of this research were verbal and non-verbal texts. The data were collected from some photograps of visually manipulated commercial advertisements from billboards that recently taken from several streets in Medan. It was collected by applying documentary technique in which only the data that support the research questions were taken. The researcher used Miles, Huberman and Saldana (2014) to analyze the data. There are three main components namely data condensation, data display, drawing and verifying conclusions.

\section{FINDINGS AND DISCUSSION}

\section{Inventory of Semiotic Meaning}

After analyzing the data it was found that there were two broad categorize of meaning. They are related to verbal and non-verbal aspects. The relation between the verbal and non-verbal expression were grouped into: the verbal expression elaborate the non-verbal (visual) expression, the verbal expression manipulate the non-verbal (visual) expression, the nonverbal (visual) expression manipulate the verbal expression, the verbal expression exageratte the non-verbal (visual) expression.

\section{The Realization of Verbal Text and Non-Verbal Text in Cigarette Advertisement Text}

In this study, there were two realizations of semiotic meaning in verbal text and non-verbal text. It can be seen as the data analysis below:

\section{DATA 1 DJARUM SUPER}

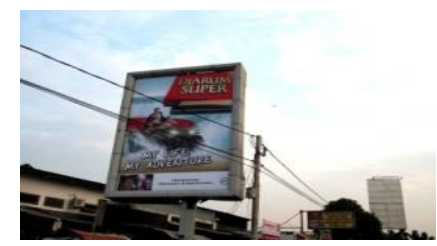

\section{Verbal Analysis}

My Life, My Adventure

12 Collectible Series

Available 11, 12, 13

This cigarette advertisement uses the slogan "My life, My adventure". This is a positive noun phrase that give a prestige, an impression/performance, to the reader or smoker 
by presenting personal pronoun "my". The pronoun seek to address the smoker's directly and personally. When people are addresses individually rather than as part of mass audience it is considered highly valued.

\section{Non-Verbal Analysis}

The representation of participants in the image are three men. There are four conceptual symbolic suggestive process where three men who are adventuring by a car. They were riding their car through the river/water. It seems that they are very excited and enjoy while on their way even though they are passing the challenges that exist in front of them.

However, there is no pictorial detail in the image for it has an aabstract representation (cigarette) rather than a concrete one which should be relevant to the advertised product. In other words, instead of depicting the real picture of cigarette, the advertiser used the activity of the human like doing an adventure that can indicates the smoker's life by using the product. The eye level angle costruct a balance of power between the viewer and advertiser; it is indicated with the represented participant being shot vertcally. While the medium shot represents a normal social distance, without any significant intimacy for the whole figure of the represented participants can be viewed with spaced arround it. Therefore, the viewer has the power to choose the product. In the other words, there is no force.

The salience used in this advertisement is red, blue, and white color. The color blue give peace to the viewer/smoker. The illumination of image shows full representation of lights and shade as the light comes from the front and it causes shadow at the represented participants, as if it looks like real. Thus, the color and such salience are not only as a meant of information but also attact the viewer's attention and encourage them to notice the advertisement. The information value of the image is indicated from two things. One of them is the right side, the New, where Djarum Super as a brand name, the text 'my life my adventure' as a slogan. The other one is the left side of the riding car, the Given, where the advertiser indicates the the life of smoker's life which same like the adventure event.

\section{DATA 2: DUNHILL MILD}

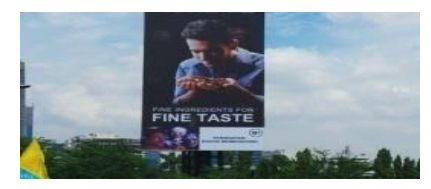

\section{Verbal Analysis}

Fine Ingredients for Fine Taste 
The verbal text in this advertisement is "Fine Ingredients for Fine Taste". This is as the slogan which is it categorize a a verbless clause. The use of positive adjective "Fine" give an impression that the product has high quality and may promise prestigious state of emotion for those who try/buy it.

\section{Non-Verbal Analysis}

The represented participant in the image is a man who is clutching spices such as cloves, cinnamons, tobaccos, etc. The conceptual symbolic suggestive process where spices (cloves, cinnamons, tobaccos) can give smoker high quality and enjoyment if the smoker buys it as in fact in their slogan "Fine ingredients for Fine taste". It is important to note that in this particular advertisement the product of the company is absent. Therefore, the focus is not only on the selling cloves, cinnamons, etc, but also to make sure that the ingredients that the product has good.

There is no direct gaze or eye contact from the repreented participant to viewer, so the image is referred as an offer for the represented participants of the image are as item of information, object of contemplation and impersonality as if they were on display that observed by viewer in terms of what brand of the cigarette that offers a better design and quality the product to smoker. The social distance of the image is medium as it is shot from a close distance. So it is viewed as a full picture of what the adviser mean to represent the cigarette product by presenting a man and some ingredients of cigarette.

The perspective of the image is frontal angle which focuses on involvement between the viewer and the represented participant as if what we see from the image implies as part of our world:the good ingredients of the cigarette and interaction with the smoker who taste it with the good taste. The image illumination shows full representation of lights and shades as the light comes from above. The salience factors shown in the image are the size large of the spices (ingredients of the cigarette) and also the image of a man. The information valued are indicated by Given (left) and New (Right). The Position of the spices and the man on the right which referred as "New" meaning the information presented is not yet know that warrants special attention from the viewers/smokers about the taste of the cigarette.

\section{DATA 3 GG MILD}

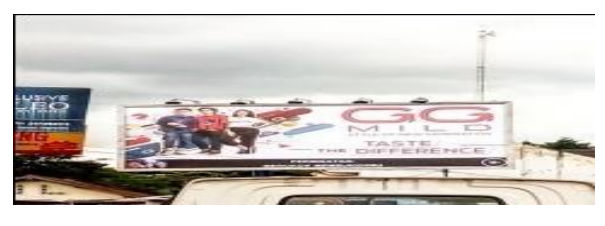




\section{Verbal Analysis}

\section{Style of New Generation}

Taste the Difference

\section{Get Your Limited Edition Pack}

The verbal text in this advertisements are the "Style of New Generation" and "Taste the Difference". This advertisement uses positive noun phrase that give a prestige, an impression/performance, to the reader or smoker by presenting noun phrases "new generation". The noun seek to address the smoker's directly and personally which means addressee/smoker is the young generation.

The slogan "taste the difference" is imperative type or direct command to persuade the smoker with the good impression of the product which it is also has good taste and difference from another. While the headline "Get Your Limited Edition Pack" is also imperative sentence by presenting word "get". Here, advertiser also use personal pronoun" you" to address the smoker's directly and personally.

\section{Non-Verbal Analysis}

The represented participants in the image is Two young men and one young girl. They look so happy, and enjoy expressed by their smile. Their style also describe that they have the unique style. The circumtance of the image is such colors as a background which is indicates for full spirit and fantastic fells. The conceptual representation of the image is symbolic attribute structure, which symbolized young generation's picture who has unique styles like using a sweater, using a jeans jacket and T-shirt.

The eye level angle costruct a balance of power between the viewer and advertiser; it is indicated with the represented participant being shot vertcally. While the medium shot represents a normal social distance, without any significant intimacy for the whole figure of the represented participants can be viewed with spaced arround it. Therefore, the viewer has the power to choose the product. In the other words, there is no force.

The salience of the image is shown by the large of GG Mild and the slogan text on the right side, especially words "taste" and "difference". It implies that the advertiser persuading smoker to do something "buy" and informing that the product is different from another. The image illumination shows full representation of light and shade as light comes from above and it causes shadow at the represented participants, as it looks like real. Thus, they are able to capture the viewer's attention and encourage them to notice the advertisement. 
The information values are indicated by Given (left) and New (right). The position of the young generation picture on the left which referred as "Given" meaning the things or information that presented are already known and familiar to the viewer and they are part of culture. The position of the brand "GG Mild", the slogan "Style of new Generation", and "Taste the difference" on the right which refers as "New" meaning the information presented is not yet know that has special attention from viewers. So, it explains what new product is like.

DATA 4: LA BOLD

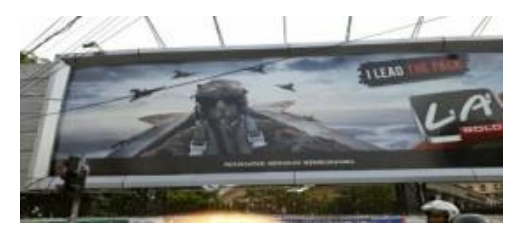

\section{Verbal Analysis}

'I Lead the Pack'

In this advertisement, the advertiser uses pnoun "I" seek to address the smoker's directly and personally. When people are addresses individually rather than as part of mass audience it is considered highly valued which is to establish the strong relationship between presenter/advertiser and the viewesr/smokers. Advertiser also uses technical or creative word to attract smokers such as "the pack" that refers to the pack of the cigarette in order to impress the consumer. The pack here that means a bundle arrange for convenience.

\section{Non-Verbal Analysis}

This picture describes that the man who is riding his fighter. In this picture, it can be seen that the man is followed by some fighter behind him. It looks like he is a learder for another fighter. He looks so brave. Through the picture, the advertiser assumes that every people who are smoke with this product, the smoker can be like a soldier who fights and can be as a leader in his war squad.

\section{DATA 5: MARLBORO}

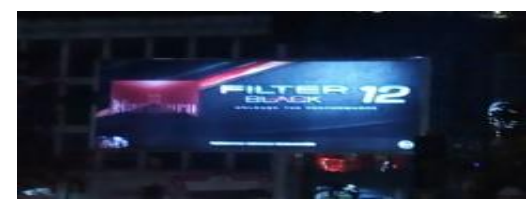

\section{Verbal Analysis}

Filter Black 12 


\section{Unleash the Performances}

In this advertisement, there are two verbal texts: "Filter Black 12" and "Unleash the Performances". Both of them are declarative sentencefor conveying information to smoker of the quality and quantity of the product it may bring. In the phrase "Filtered blak 12", advertiser tried to inform that their product has a good taste by providing words "filter and black" and number " 12 " indicates that the cigarette has 12 stems in a pack.

The phrase "Unleash the Performances" is an imperative type or direct comman to persuade reader to buy or take certain action. The collocation "the performances" means to express ideological opinions about things and events in the cigarette advertisement.

\section{Non-Verbal Analysis}

In this advertisement, advertiser uses the word 'filter black' assumes this kind of cigarette is good. It has good ingredients inside the cigarette. The advertiser also uses the words' unleash the performance' in order to ask smokers to keep enjoy with their life and not to be affraid to show their talent, their hobby, and others creativity that they can create in their life.

\section{The Reason why Semiotic Meaning coded in the Cigarette Advertisement}

In describing the context of semiotic meaning in cigarette advertisement in this case was context of situation. The context of semiotic meaning in cigarette advertisement in terms of notion of field, tenor, and mode. The rhetorical mode in this advertisement was persuasive mode. Persuasive means able to cause people to do or believe something: able to persuade people. An advertisement is used to persuade people to do or believe of what the advertiser say or do. Based on the result of data analysis, the findings of this study are formulated as the following:

1) There are two broad categorizes of meaning. They are related to verbal and non-verbal aspects. The relation between the verbal and non-verbal expression are grouped into: the verbal expression elaborate the non-verbal (visual) expression, the verbal expression manipulate the non-verbal (visual) expression, the non-verbal (visual) expression manipulate the verbal expression, the verbal expression exageratte the non-verbal (visual) expression.

2) Relating to the linguistic aspect occur in 20 cigarette advertisement texts, they make the major use of declarative sentence that are found on 11 texts. Then, by using 8 imperative and 8 adjective. Next, by using 6 disjunctive and 6 noun/pronoun. The major occurances of 
declarative sentence in the cigarette advertisement are used to manipulate potential buyer/smoker by conveying information or suggestion to the smoker about the quality of the product or the profit it may bring whether it is true or not.

Relating to image/picture in the advertisement, the advertiser makes the major use of visual features such as calor saturation, symbolic structure representation, offering gaze, the use of large size, logo, slogan. Then followed by one more typically manipulative visual semiotic feature such as illumination (the creation of lights and shade). All of it components potentially able to persuade the smoker to but the product.

3) Various social context are discoverd in the advertisement such as promoting the standar of quality, quantity, creative, enjoying the life. The standard idea about how particular mode like young and old man should be differentiated based on the conventional values in society. When it comes to what types of product that is competible to them for example the representation of the picture of man who are playing with some instrument like guitar, drum, piano and also playing the skateboard. Cigarette advertisement also hide threats with persuasive expression and colorful meaning for commercial purposes.

\section{CONCLUSION}

1) In this research, the researcher found that the advertisement used the relation between the verbal and non-verbal expression.

2) The cigarette advertisement texts were dominated by using declarative sentence in the verbal text which mean that the cigarette advertisement text has purpose to persuade smoker by adding characteristics of the product like using adjective good, fine, modern, etc. While in non-verbal text, the advertisers use some picture with the sense of power, energy, excitement, strength, seductive, passionate, aggressive, hardship, fear, confident, conservative, relaxing, simple, friendly, joyful, light, free, vigorous, sunny, and happines. The advertiser makes the major use of visual features Then followed by one more typically manipulative visual semiotic feature such as illumination (the creation of lights and shade). All of it components were potentially able to persuade the smoker to but the product.

3) The use of semiotic meaning (sign ) is related to the context of situation. The finding showed that the used of semiotic meaning (sign) is increased when the advertisers conveyed the information to the custumer when they are trying to indicate to the custumer to what extend they align themselves with evidence they are informing in their opinion since one the purpose of the advertisement is to persuade the customer. 


\section{REFERENCES}

Fairclough, N., 1995, Critical Discourse Analysis, London: Longman.

Fairclough, N. 2001. Critical discourse analysis as a method in social scientific research. In:

R. Wodak \& M. Meyer (Eds.), Methods of critical discourse analysis (pp.121138). London: SAGE Publications.

Kress, G. 2003. Multimodality-a Social Semiotic Approach to Contemporary Communication. New York: Routledge.

Kress, G. \& Van Leeuwen, T. 2006. Reading Images. London: Routledge.

Krefting. L. 1991. Rigor in Qualitative Research: the Assessment of Trustworthiness. The American Journal of Occupational Therapy, 43(3), 214.

Lincoln, Y. S. \& Guba, E. G. 1985. Naturalistic Inquiry. California: Sage Publication, Inc.

Meyer, M. 2001. Between Theory, Method, and Politics: Positioning of the Approaches to CDA. In: R. Wodak \& M. Meyer (Eds.), Methods of Critical Discourse Analysis (pp.14-31). London: SAGE Publications.

Miles, B. M,. Michael, H. \& Johnny, S. 2014. Qualitative Data Analysis: a Method of Sourcebook: $3^{\text {rd }}$ Edition. USA. SAGE.

Wodak, R. 1999. Critical Discourse Analysis at the End of the 20th Century. Research on Language and Social Interaction, 32, 1 \& 2, 185-193. 